\title{
Dissimilar Effects of Tolterodine on Detrusor Overactivity in Awake Rats with Chemical Cystitis and Partial Bladder Outlet Obstruction
}

\author{
Long-Hu Jin, Chang-Shin Park ${ }^{1}$, Hwa-Yeon Shin, Sang-Min Yoon, Tack Lee \\ Departments of Urology and ${ }^{1}$ Pharmacology, Inha University College of Medicine, Incheon, Korea
}

\begin{abstract}
Purpose: We investigated bladder function, with a special focus on nonvoiding contractions (NVCs), in awake rats with chronic chemical cystitis and bladder outlet obstruction (BOO) by use of simultaneous registrations of intravesical and intraabdominal pressures. In addition, we tested the effects of tolterodine on the NVCs in these models.

Methods: A total of 20 female Sprague-Dawley rats were used in this study. In eight rats, chemical cystitis was induced by intravesical instillation of $\mathrm{HCl}$. Twelve rats were subjected to sham instillations or partial BOO. Four weeks after intravesical instillation or 2 weeks after partial BOO, cystometrograms were obtained by use of simultaneous recording of intravesical and intraabdominal pressure in all unanesthetized, unrestrained rats in metabolic cages.

Results: A total of 17 rats survived. In the rats with acute injury by $\mathrm{HCl}, 50 \%$ showed detrusor overactivity (DO), which was not seen in the sham group. The cystitis group had lower DO pressure without a difference in DO frequency compared with the BOO group. After the administration of tolterodine, the cystitis group showed no difference in DO frequency or pressure, whereas the BOO group showed decreased values for both parameters.

Conclusions: Our study showed that toleterodine produced no effect on DO during the filling phase in rats with chronic chemical cystitisbut decreased the frequency and pressure of DO in rats with BOO. Clinically, studies are needed to improve the treatment effect of anticholinergic drugs ininterstitial cystitis patients with overactive bladder.
\end{abstract}

Keywords: Overactive urinary bladder; Urodynamics; Rats

\section{INTRODUCTION}

Interstitial cystitis (IC) is one of the most commonly seen chronic inflammatory diseases of the urinary bladderand is characterized by a wide range of symptoms, including pelvic or lower abdominal pain and irritative voiding symptoms such as urinary frequency, urgency, and nocturia [1-3]. Overlapping patterns of lower urinary tract symptoms and pelvic pain are common in patients with IC [4], and these overlapping patterns create challenges for clinical research and practice and warrant further investigation of their causes, diagnosis, and effective treatments.
The conditions of IC and overactive bladder (OAB) share irritative voiding symptoms $[1,4]$. Theoretically, then, anticholinergic drugs could be considered to provide an alternate mechanism of action, which could decrease these embarrassing symptoms. In practice, however, many studies have shown that most IC patients do not respond appropriately to treatment with anticholinergic medications, whereas $\mathrm{OAB}$ patients with benign prostatic hyperplasiarespond well $[5,6]$. Thus, we need to clarify the urodynamic findings and mechanismsin these two experimental conditions in animal models.

The OAB syndrome is defined as urinary urgency, with or without urge incontinence, that is usually accompanied by uri-
Corresponding author: Tack Lee

Department of Urology, Inha University College of Medicine by BK 21 Project, Sinheung-dong 3-ga, Jung-gu, Incheon 400-711, Korea

Tel: +82-32-890-3448 / Fax: +82-32-890-3097 / E-mail address: lt11@inha.ac.kr Submitted: August 30, 2011 / Accepted after revision: September 26, 2011
This is an Open Access article distributed under the terms of the Creative Commons Attribution Non-Commercial License (http://creativecommons.org/licenses/by-nc/3.0/) which permits unrestricted non-commercial use, distribution, and reproduction in any medium, provided the original work is properly cited. 
nary frequency and nocturia [7]. In other words, this urgency is regarded as a core symptom in diagnosis, which may correspond to detrusor overactivity (DO) during the filling phase in human urodynamic study $[7,8]$. Assuming that nonvoiding contractions (NVCs) interpreted on the basis of simultaneous changes in intraabdominal pressure (IAP) in an animal model can be used as a substitute parameter for DO in humans, the rat model of chemical cystitis and bladder outlet obstruction (BOO) may allow us to study the pathophysiology of different responsesto anticholinergic drugs.

In this study, we investigated bladder function, with a special focus on the NVCs, in awake rats with chemical cystitis and BOO by use of simultaneous registrations of intravesical and intraabdominal pressures. In addition, we tested the effects of tolterodine on the NVCs in these models.

\section{MATERIALS AND METHODS}

\section{Animals and Study Design}

A total of 20 female Sprague-Dawley rats (250 to $295 \mathrm{~g}$; Orient Bio Inc., Seongnam, Korea) were used in this study. All experimental animals and procedures were performed in accordance with the Guide for the Care and Use of Laboratory Animals of the National Institutes of Health and were approved by the local animal ethics committee. The rats were maintained under standard laboratory conditions with 12:12 hours light:dark cycles and free access to food pellets and tap water. In eight rats, chemical cystitis was induced by intravesical instillation of $\mathrm{HCl}$. Twelve rats were subjected to sham instillations or partial BOO. Four weeks after intravesical instillation or 2 weeks after partial $\mathrm{BOO}$, cystometrograms were obtained in all unanesthetized, unrestrained rats in metabolic cages.

\section{Induction of Cystitis}

Rats were anesthetized with intraperitoneal ketamine (Ketamine, Yuhan Corp., Seoul, Korea; 75 mg/kg) and xylazine (Rompun, Bayer Korea Ltd., Seoul, Korea; 15 mg/kg intraperitoneally). Cystitis was induced by the intravesical instillation of $0.5 \mathrm{~mL}$ of $0.4 \mathrm{~N} \mathrm{HCl}$ [9]. Through an abdominal incision under anesthesia, a 24-gauge needle with syringe was inserted into the dome of the bladder. After all of the urine was aspirated, $\mathrm{HCl}$ was instilled into the bladder lumen for 90 seconds. The same volume of normal saline was instilled in the sham group.

\section{Procedures for Intravesical, Intraabdominal, and Intravenous Catheter Implantation}

Three days before cystometry, catheterizations for simultaneous recordings of intravesical pressure (IVP) and IAP were performed as described previously [10]. Briefly, after anesthesia, a polyethylene catheter (PE-50, Becton Dickinson, Parsipanny, NJ, USA) with a cuff was implanted into the dome of the bladder through an abdominal incision. To record IAP, an abdominal balloon (Latex, Dawoo Medical, Incheon, Korea) around the cuff of a catheter tip was placed proximal to the bladder and was tied to another catheter with silk tie. To deliver the anticholinergic agent during awake cystometry, a polyethylene catheter (PE-10) was inserted into a femoral vein, which was filled with heparinized (30 IU) saline. These catheters were then tunnelled through the subcutaneous space and exited the back of the animal and were anchored to the skin of the back. After surgery, each rat was caged individually and maintained in the same manner.

\section{Functional Evaluation}

Cystometrograms were obtained in unanesthetized, unrestrained rats in metabolic cages. The indwelling catheter to the bladder was connected to a two-way valve that was connected via a T-tube to a pressure transducer (Research Grade Blood Pressure Transducer, Harvard Apparatus, Holliston, MA, USA) and a microinjection pump (PHD22/2000 pump, Harvard Apparatus). Another indwelling catheter connected to a fluid-filled abdominal balloon was connected to another pressure transducer to record the IAP. Micturition volumes were recorded by means of a fluid collector connected to a force displacement transducer (Research Grade Isometric Transducer; Harvard Apparatus). IVP, IAP, and micturition volumes were recorded continuously with Acq Knowledge 3.8.1 software and an MP150 data acquisition system (BIOPAC Systems, Goleta, CA, USA) at a sampling rate of $50 \mathrm{~Hz}$. The mean values from three reproducible micturition cycles were used for evaluation. IAP was defined as the recorded balloon pressure minus the lowest balloon pressure in each voiding cycle. Detrusor pressure (DP) was defined as IVP minus IAP. The IVP rises during the filling phase were defined as increments of IVP that exceeded $2 \mathrm{~cm}$ $\mathrm{H}_{2} \mathrm{O}$ from baseline, which was interpreted as DO if occurring without simultaneous similar changes in IAP and was interpretedas abdominal straining if occurring with simultaneous similar changes in IAP. 


\section{Investigated Urodynamic Parameters}

Pressure-and volume-related parameters

These included basal pressure (BP; the lowest bladder pressure during filling), threshold pressure (TP; bladder pressure immediately before micturition), maximum pressure (MP; maximum bladder pressure during the micturition cycle), micturition volume (MV; volume of expelled urine), residual volume (RV; remaining urine after voiding), bladder capacity (BC; $\mathrm{MV}+\mathrm{RV})$, and micturition interval (MI; intervals between micturition contractions).

\section{DO-related parameters during the filling phase}

These included time of filling phase (interval from the initiation of infusion through the tube and the point immediately before the initiation of micturition), frequency of abdominal strainingper minute, frequency of DO per minute, and increased amplitude from base to peak of DO spike as IVP. These frequencies were calculated on the basis of the time of filling phase. After cystometry, the animals were sacrificed by cervical dislocation. The bladder and urethra were removed en bloc and separated at the level of the bladder neck, and the bladder was weighed.

\section{Administration of Drug}

During cystometry, room temperature saline was infused into the bladder at a rate of $10 \mathrm{~mL} \cdot \mathrm{h}^{-1}$. Micturitions during intravesical saline infusion served as baseline values. After $0.2 \mathrm{~mL}$ tolterodine $(0.3 \mathrm{mg} / \mathrm{kg})$ was injected intravenously, there was a 30-minute observation period.

\section{Statistical Analysis}

The results are given as mean values \pm standard errors of the mean (SEMs). Normal distributions were confirmed by the Shapiro-Wilks' W test. One-way analysis of variance with Tukey's test was used to detect differencesin urodynamic parameters among the sham, cystitis, and BOO groups before drug administration, and paired testswere used to compare these parameters between before and after drug administration in the same group. All analyses were performed with GraphPad Prism, ver. 5.03, 2009 (GraphPad Software, San Diego, CA, USA). Statistical significance was considered at $\mathrm{P}<0.05, \mathrm{P}<0.01$, and $\mathrm{P}<0.001$. All calculations were made based on $n$, which denoted the number of animals.

\section{RESULTS}

\section{Comparison of Body Weights and the Ratio of Bladder to Body Weight}

There wereno significant differences among the sham (266.7 \pm $6.7 \mathrm{~g})$, cystitis $(281.7 \pm 4.4 \mathrm{~g})$, and $\mathrm{BOO}(261.0 \pm 6.0 \mathrm{~g})$ groups in body weight at 2 weeks after intravesical instillations or obstruction surgery. However, the ratio of urinary bladder weight to body weight significantly increased in the BOO group $(1.00 \pm 0.12)$ compared with the sham $(0.43 \pm 0.02)$ andcystitis $(0.45 \pm 0.03)$ groups. There was no significant difference between the sham and cystitis groups in that ratio (Fig. 1A).

\section{Comparison of Pressure-Related Parameters among the Three Groups and Effects of Tolterodine}

The BOO group showed increased $\mathrm{BP}$ and $\mathrm{TP}$ by DP compared
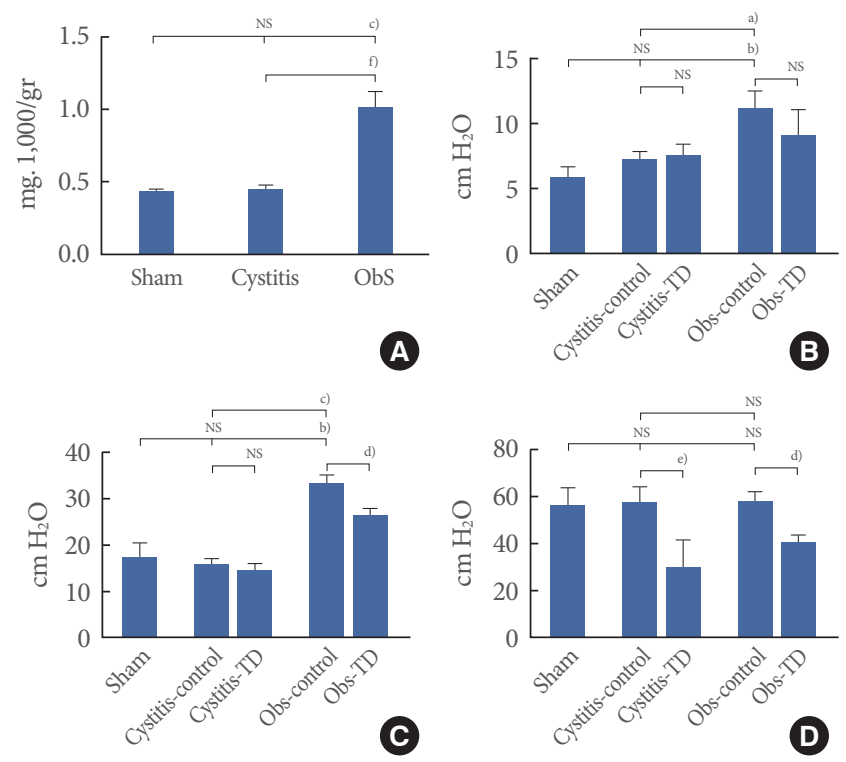

Fig. 1. The ratio of bladder weight (mg) to body weight (g) (A) and the effects of tolterodine on pressure-related parameters in rats in the sham, chemical cystitis, and bladder outlet obstruction groups. (B) Basal pressure. (C) Threshold pressure. (D) Maximal pressure. Cystitis-control, micturition cycles without medication in the cystitis group; Cystitis-TD, micturition cycles after theadministration of tolterodine ( $3 \mathrm{mg} / \mathrm{kg}$ i.v.); Obs-control, micturition cycles without medication in the bladder outlet obstruction group; Obs-TD, micturition cycles after the administration of tolterodine ( $3 \mathrm{mg} / \mathrm{kg}$ i.v.); NS, not significant. Results are expressed as the mean \pm standard error of the mean. ${ }^{\text {a) }} \mathrm{P}$ $<0.05 .{ }^{\text {b) }} \mathrm{P}<0.01 .{ }^{\mathrm{c}} \mathrm{P}<0.001$ (unpaired Student's t-test); ${ }^{\mathrm{d}} \mathrm{P}$ $<0.05 .{ }^{\mathrm{e})} \mathrm{P}<0.01 .{ }^{\mathrm{f}} \mathrm{P}<0.001$ (paired Student's t-test). 
with thecystitis and sham groups, but there were no significant differences in those values between the cystitis and sham groups. There were no significant differences in MP by DP among the three groups (Fig. 1B-D).

After the administration of tolterodine, the cystitis and BOO groups showed decreased MP by DP compared withthe values before medication (Fig. 1D). The BOO group showed decreased TP by DP, whereas the cystitis group showed no changes in this variable after medication. BP by DP did not change significantly in either group with medication (Fig. 1B, C).

\section{Comparison of Volume-Related Parameters among the Three Groups and Effects of Tolterodine}

There were no significant differences in any volume-related parameters before medication, including $\mathrm{BC}, \mathrm{MV}, \mathrm{RV}$, and $\mathrm{MI}$, among thesham, cystitis, and BOO groups (Fig. 2).

There were no significant changes in $\mathrm{BC}$ or $\mathrm{MI}$ after the administration of tolterodine in either cystitisor $\mathrm{BOO}$ rats. Also,
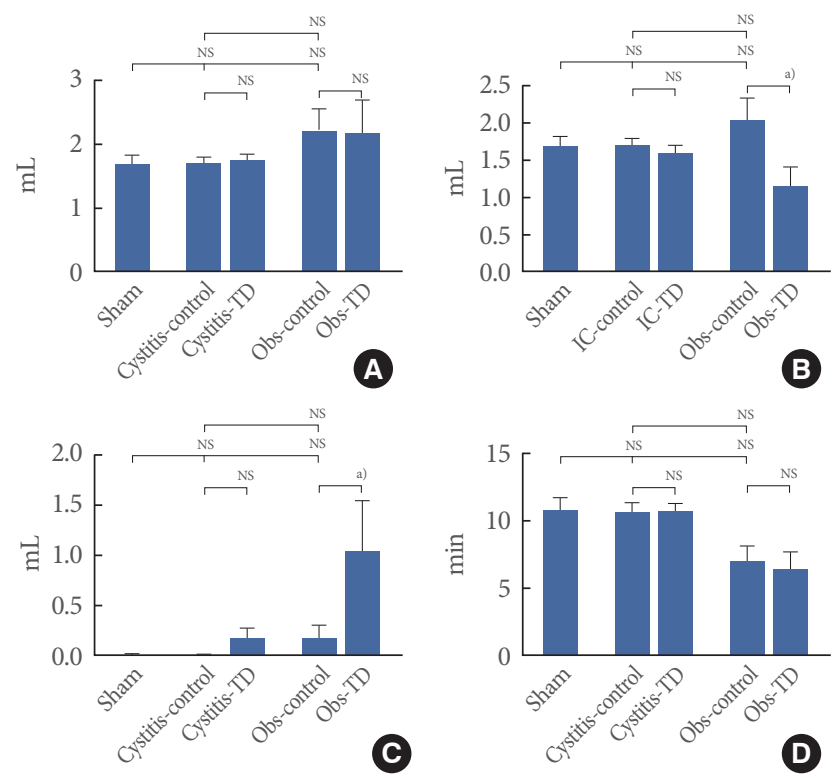

Fig. 2. The effects of tolterodine on volume-related parameters in rats in the sham, chemical cystitis, and bladder outlet obstruction groups. (A) Bladder capacity. (B) Micturition volume. (C) Residual volume. (D) Micturition interval. Cystitis-control, micturition cycles without medication in the cystitis group; Cystitis-TD, micturition cycles after theadministration of tolterodine (3 mg/kg i.v.); Obs-control, micturition cycles without medication in the bladder outlet obstruction group; Obs-TD, micturition cycles after theadministration of tolterodine ( $3 \mathrm{mg} /$ kg i.v.); NS, not significant. Results are expressed as the mean \pm standard error of the mean. ${ }^{\text {a) }} \mathrm{P}<0.05$ (paired Student's t-test). the cystitis group showed no changes in MV after medication, whereas values decreased in the BOO group. As for RV, the cystitis group also showed no changes despite higher values in the BOO group after medication (Fig. 2).

\section{Comparison of DO-Related Parameters between the Sham and Cystitis Groups and Effects of Toleterodine}

The cystitis group showed the DO in 50\% (3 of 6 rats), which was not seen in the sham group. Before theadministration of tolterodine, the cystitis group showed lower DO pressure, without a difference in $\mathrm{DO}$ frequency, than did the $\mathrm{BOO}$ group. After the administration, the cystitis group showed no difference in DO frequency orpressure, although the BOO group showed decreased values forboth parameters (Figs. 3, 4).

\section{DISCUSSION}

Our study showed that tolterodine had no significant effect on
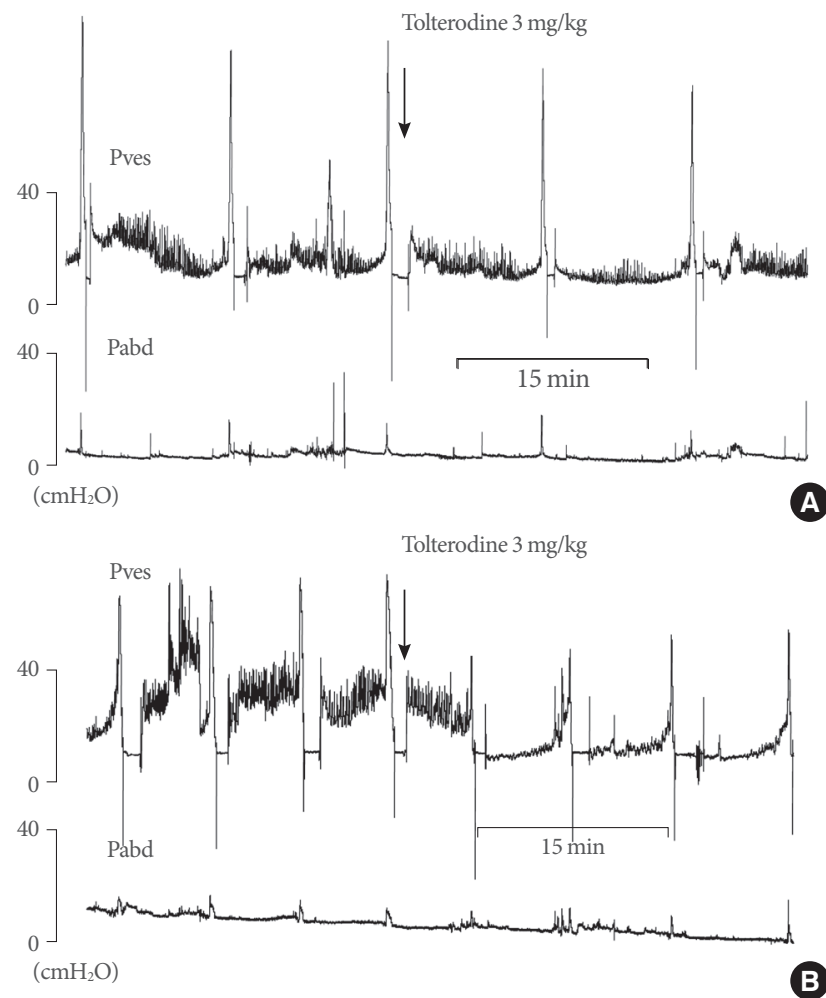

Fig. 3. Representative tracings of intravesical and intraabdominal pressures showing total micturition cycles, before and after the administration of tolterodine ( $3 \mathrm{mg} / \mathrm{kg}$ i.v.), in rats with chemical cystitis and bladder outlet obstruction (BOO). (A) Chemical cystitis model. (B) BOO model. Pves, intravesical pressure; Pabd, intraabdominal pressure. 

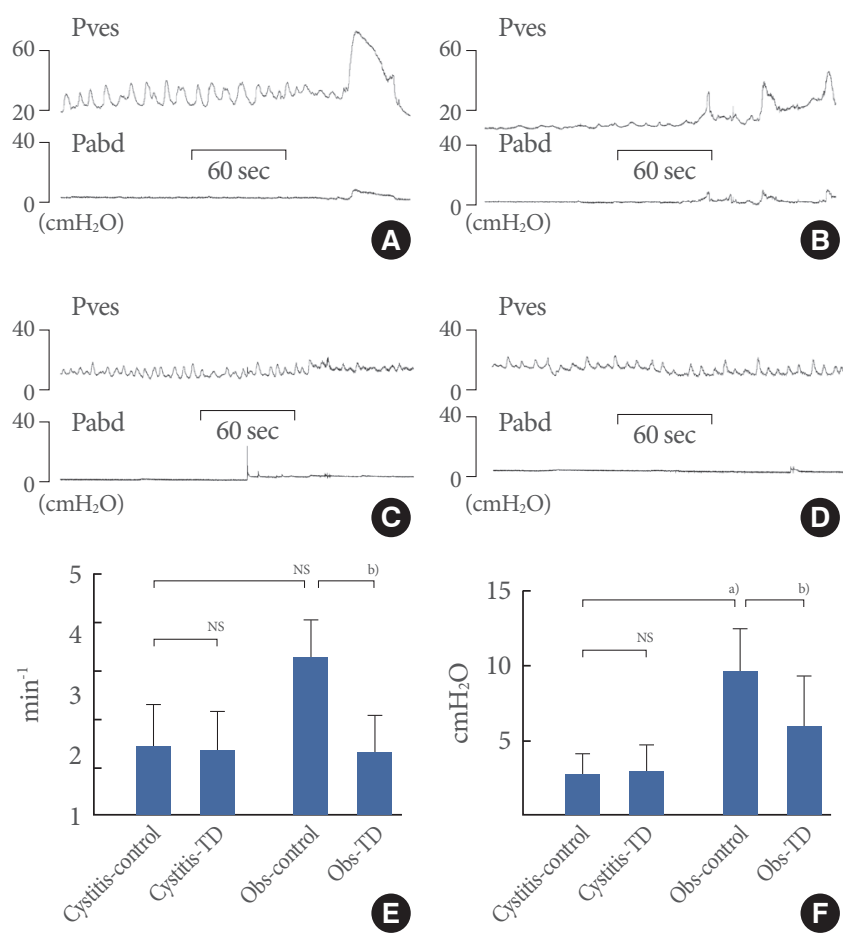

Fig. 4. Representative tracings of intravesical and intraabdominal pressures showing minute changes in detrusor overactivity (DO) before and after the administration of tolterodine (3 mg/ $\mathrm{kg}$ i.v.) in rats with chemical cystitis and bladder outlet obstruction (BOO). (A) Chemical cystitis model before medication. (B) Chemical cystitis model after injection. (C) BOO model before medication. (D) BOO model after injection. (E) Changes in DO frequency. (F) Changes in DO pressure. Cystitis-control, micturition cycles without medication in the cystitis group; CystitisTD, micturition cycles after the administration of tolterodine; Obs-control, micturition cycles without medication in the bladder outlet obstruction group; Obs-TD, micturition cycles after the administration of tolterodine; NS, not significant; Pves, intravesical pressure; Pabd, intraabdominal pressure. Results are expressed as the mean \pm standard error of the mean. ${ }^{\text {a) }} \mathrm{P}<0.05$ (unpaired Student's t-test); ${ }^{\text {b) }} \mathrm{P}<0.05$ (paired Student's t-test).

urodynamic parameters, including pressure/volume and DOrelated variables, except MP in a chronic IC model. Only MP showed a decrease with treatment without changes in DO frequency or pressure. This suggests that toleterodine may have an unfavorable effect on voiding without any effect on DO during the filling phase. In the BOO model, however, the drug resulted in adecrease inboth frequency and pressure of DO and some changes in pressure/volume parameters. The anticholinergic drug showed some effects on both the filling and voiding phases. IC is a chronic inflammatory disease of the bladder [1], but its etiology and pathogenesis remain obscure. It is characterized by irritative voiding symptoms and vague pain related to the bladder and shareswith $\mathrm{OAB}$ syndromeurinary urgency as a key symptom in diagnosis $[1,4]$. The urgency symptom is known to be correlated with the DO, which is defined as a urodynamic observation of involuntary detrusor contractions during the bladder-filling phase $[7,8]$. About $15 \%$ of patients with IC have been reported to have spontaneous DO as measured by urodynamic testing [11]. The treatment of this DO has incorporated the application of anticholinergic drugs, but many clinical reports have shownthat these drugs are ineffective in many patients with IC $[5,6]$. There havebeen few reports directly showing the effects of anticholinergic drugs on urodynamic results, including objective report of DO in an awake animal IC model. According to our results, DO was not affected by the anticholinergic drug in the IC model, unlike in the BOO model. Such study to find the cause of the ineffectiveness in an animal model is important to improve our understanding of these drugs and to find possible alternative treatments.

The uncertainty over the benefit of anticholinergic drugs in IC appears to stem from two separate factors of unclear diagnostic criteria and etiology or pathophysiology. In the diagnosis of IC, after ruling out other obvious pathology and overlapping syndromes through an extensive and specific series of tests [12], the exclusion of other often confused diseases is still encouraged, because there are no definitive diagnostic tests. Since the National Institute for Diabetes and Diseases of the Kidney (NIDDK) reported the diagnostic criteria for IC in 1990 [13], the definitions and terminology have undergone some changes. Different organizations have proposed different terms, such as painful bladder syndrome or bladder pain syndrome $[14,15]$. Enigmatic pain related to the bladder was emphasized more than the other symptoms in these reports $[3,14]$. However, because these small changes in diagnostic criteria can result in noticeably different patient groups being studied, we need to unify the terms or definitions and focus on how to delineate the patients accurately by use of accurate diagnostic criteria internationally. The heterogeneous characteristics of IC subgroups could be the starting obstacle in finding a solution to the ineffectiveness of anticholinergic drugs. It will be important to find a subgroup of IC patients who respond well to this drug or to determine how to use this drug as a palliative agent.

Second, several decades of research on IC has revealed that inflammation, as determined by the presence of cytokines, chemokines and mast cells, may have vital role in the pathogenesis 
of this disease $[16,17]$. Although it is suggested that increased permeability of or neuropathic pain in the bladder mucosa results in $\mathrm{OAB}$ through the activation of afferent reflex circuitry [18-20], our limited understanding of the fundamental mechanisms causing $\mathrm{OAB}$ in IC has continued to frustrate efforts aimed at its treatment. The detailed mechanism needs further elucidation.

Our proposed model of chronic chemical cystitis showed the characteristics of $\mathrm{OAB}$ in view of DO-related parameters. Our modelshowed DO in $50 \%$ of the rats, even with no increased $\mathrm{BP}$, TP, or MP and no decreased BC, MV, RV, or MI compared with the control rats. These animals at 4 weeks did not show differences compared with animals at 2 weeks in our previous study [21]. Our model of BOO also showed characteristics similar to the results of previous studies with $\mathrm{BOO}$. The $\mathrm{BOO}$ rats showed an increased ratio of bladder to body weight, increased $\mathrm{BP}$, and increased TP, but no increases in BC, MI, or RV. After theadministration of tolterodine, the BOO model showed a decrease inTP, MP, and DO pressure/frequency and an increase in $\mathrm{RV}$, corresponding to the original effects of that drug.

In the obstructed bladder, supersensitivity of the detrusor muscle secondary to denervation was found to play a predominant pathophysiologic role in OAB [22,23]. This has been suggested to be caused by bladder overdistention resulting from BOO [24]. Clinically, DO in men with OAB responds well to antimuscarinics, which is similar the results of our study [25]. An anticholinergic agent is a substance that blocks the acetylcholine of a neurotransmitter by competitive inhibition in neurons of the central and peripheral nervous system. This is known to reduce the effects mediated by acetylcholine on muscarinic receptors in those neurons [26]. We suspect that the different response between $\mathrm{BOO}$ and IC is caused by the basic difference in the pathophysiologic characteristics of the bladder in these two conditions, which needs to be elucidated further in future studies. Clinically, we also need further study to improve the treatment effect of anticholinergic drugs inIC patients with $\mathrm{OAB}$.

\section{CONFLICT OF INTEREST}

No potential conflict of interest relevant to this article was reported.

\section{ACKNOWLEDGEMENTS}

This study was supported by grants from the Korea Healthcare technology R\&D Project, Ministry of Health \& Welfare, Republic of Korea (A090715-1002-0000100).

\section{REFERENCES}

1. Gillenwater JY, Wein AJ. Summary of the National Institute of Arthritis, Diabetes, Digestive and Kidney Diseases Workshop on Interstitial Cystitis, National Institutes of Health, Bethesda, Maryland, August 28-29, 1987. J Urol 1988;140:203-6.

2. Ibrahim IA, Diokno AC, Killinger KA, Carrico DJ, Peters KM. Prevalence of self-reported interstitial cystitis (IC) and interstitialcystitis-like symptoms among adult women in the community. Int Urol Nephrol 2007;39:489-95.

3. Sant GR. Etiology, pathogenesis, and diagnosis of interstitial cystitis. Rev Urol 2002;4 Suppl 1:S9-S15.

4. Barry MJ, Link CL, McNaughton-Collins MF, McKinlay JB; Boston Area Community Health (BACH) Investigators. Overlap of different urological symptom complexes in a racially and ethnically diverse, community-based population of men and women. BJU Int 2008;101:45-51.

5. Minaglia S, Ozel B, Bizhang R, Mishell DR Jr. Increased prevalence of interstitial cystitis in women with detrusor overactivity refractory to anticholinergic therapy. Urology 2005;66:702-6.

6. Alhasso AA, McKinlay J, Patrick K, Stewart L. Anticholinergic drugs versus non-drug active therapies for overactive bladder syndrome in adults. Cochrane Database Syst Rev 2006;(4):CD003193.

7. Abrams P. Describing bladder storage function: overactive bladder syndrome and detrusor overactivity. Urology 2003;62(5 Suppl 2): 28-37.

8. Abrams P, Cardozo L, Fall M, Griffiths D, Rosier P, Ulmsten U, et al. The standardisation of terminology of lower urinary tract function: report from the Standardisation Sub-committee of the International Continence Society. Neurourol Urodyn 2002;21:167-78.

9. Rivas DA, Chancellor MB, Shupp-Byrne S, Shenot PJ, McHugh K, McCue P. Molecular marker for development of interstitial cystitis in rat model: isoactin gene expression. J Urol 1997;157:1937-40.

10. Lee T, Andersson KE, Streng T, Hedlund P. Simultaneous registration of intraabdominal and intravesical pressures during cystometry in conscious rats--effects of bladder outlet obstruction and intravesical PGE2. Neurourol Urodyn 2008;27:88-95.

11. Kirkemo A, Peabody M, Diokno AC, Afanasyev A, Nyberg LM Jr, Landis JR, et al. Associations among urodynamic findings and 
symptoms in women enrolled in the Interstitial Cystitis Data Base (ICDB) Study. Urology 1997;49(5A Suppl):76-80.

12. Hanno PM. Interstitial cystitis-epidemiology, diagnostic criteria, clinical markers. Rev Urol 2002;4 Suppl 1:S3-8.

13. Metts JF. Interstitial cystitis: urgency and frequency syndrome. Am Fam Physician 2001;64:1199-206.

14. El Khoudary SR, Talbott EO, Bromberger JT, Chang CC, Songer TJ, Davis EL. Severity of interstitial cystitis symptoms and quality of life in female patients. J Womens Health (Larchmt) 2009;18:1361-8.

15. Michael YL, Kawachi I, Stampfer MJ, Colditz GA, Curhan GC. Quality of life among women with interstitial cystitis. J Urol 2000; 164:423-7.

16. Desireddi NV, Campbell PL, Stern JA, Sobkoviak R, Chuai S, Shahrara S, et al. Monocyte chemoattractant protein-1 and macrophage inflammatory protein-1alpha as possible biomarkers for the chronic pelvic pain syndrome. J Urol 2008;179:1857-61.

17. Sant GR, Theoharides TC. The role of the mast cell in interstitial cystitis. Urol Clin North Am 1994;21:41-53.

18. Yoshimura N, de Groat WC. Increased excitability of afferent neurons innervating rat urinary bladder after chronic bladder inflammation. J Neurosci 1999;19:4644-53.

19. Coderre TJ, Katz J, Vaccarino AL, Melzack R. Contribution of central neuroplasticity to pathological pain: review of clinical and ex- perimental evidence. Pain 1993;52:259-85.

20. Dupont MC, Spitsbergen JM, Kim KB, Tuttle JB, Steers WD. Histological and neurotrophic changes triggered by varying models of bladder inflammation. J Urol 2001;166:1111-8.

21. Jin LH, Shin HY, Kwon YH, Park CS, Yoon SM, Lee T. Urodynamic findings in an awake chemical cystitis rat model observed by simultaneous registrations of intravesical and intraabdominal pressures. Int Neurourol J 2010;14:54-60.

22. Gosling JA, Gilpin SA, Dixon JS, Gilpin CJ. Decrease in the autonomic innervation of human detrusor muscle in outflow obstruction. J Urol 1986;136:501-4.

23. Speakman MJ, Brading AF, Gilpin CJ, Dixon JS, Gilpin SA, Gosling JA. Bladder outflow obstruction--a cause of denervation supersensitivity. J Urol 1987;138:1461-6.

24. Yokoyama O, Nagano K, Kawaguchi K, Hisazumi H. The response of the detrusor muscle to acetylcholine in patients with infravesical obstruction. Urol Res 1991;19:117-21.

25. Chapple C. Antimuscarinics in men with lower urinary tract symptoms suggestive of bladder outlet obstruction due to benign prostatic hyperplasia. Curr Opin Urol 2010;20:43-8.

26. Andersson KE, Yoshida M. Antimuscarinics and the overactive detrusor--which is the main mechanism of action? Eur Urol 2003;43: $1-5$. 